PROCEEDINGS OF THE

AMERICAN MATHEMATICAL SOCIETY

Volume 131, Number 5, Pages 1565-1572

S 0002-9939(02)06799-0

Article electronically published on October 24, 2002

\title{
GEVREY VECTORS OF MULTI-QUASI-ELLIPTIC SYSTEMS
}

\author{
CHIKH BOUZAR AND RACHID CHAILI
}

(Communicated by David S. Tartakoff)

\begin{abstract}
We show that the multi-quasi-ellipticity is a necessary and sufficient condition for the property of elliptic iterates to hold for multi-quasihomogenous differential operators.
\end{abstract}

\section{INTRODUCTION}

Let $P_{j}(x, D)=\sum_{\alpha} a_{j \alpha}(x) D^{\alpha}, j=1, \ldots, N$, henceforth denoted $\left(P_{j}\right)_{j=1}^{N}$, be linear differential operators with $C^{\infty}$ coefficients in an open subset $\Omega$ of $\mathbb{R}^{n}$.

The aim of this work is to prove the property of elliptic iterates for multi-quasielliptic systems of differential operators in generalized Gevrey spaces $G^{\mathcal{F}, s}(\Omega)$, where $\mathcal{F}$ denotes Newton's polyhedron of the system $\left(P_{j}\right)_{j=1}^{N}$. The property of elliptic iterates for the system $\left(P_{j}\right)_{j=1}^{N}$ in the generalized Gevrey classes $G^{\mathcal{F}, s}(\Omega)$ means the following inclusion:

$$
G^{s}\left(\Omega,\left(P_{j}\right)_{j=1}^{N}\right) \subset G^{\mathcal{F}, s}(\Omega) .
$$

Definition 1. Newton's polyhedron of the system $\left(P_{j}\right)_{j=1}^{N}$ at the point $x_{0} \in \Omega$, denoted $\mathcal{F}\left(x_{0}\right)$, is the convex hull of the set $\left\{\alpha \in \mathbb{N}^{n}, \exists j \in\{1, \ldots, N\} ; a_{j \alpha}\left(x_{0}\right) \neq 0\right\}$. A Newton's polyhedron $\mathcal{F}$ is said to be regular if there exists a finite set $Q(\mathcal{F}) \subset$ $\left(\mathbb{R}_{+}^{*}\right)^{n}$ such that

$$
\mathcal{F}=\bigcap_{q \in Q(\mathcal{F})}\left\{\alpha \in \mathbb{R}_{+}^{n},\langle\alpha, q\rangle \leq 1\right\}
$$

Set

$$
\begin{aligned}
k(\alpha, \mathcal{F}) & =\inf \left\{t>0, t^{-1} \alpha \in \mathcal{F}\right\}, \alpha \in \mathbb{R}_{+}^{n}, \\
\mu(\mathcal{F}) & =\max _{1 \leq j \leq n} \mu_{j}(\mathcal{F}) \\
\mu_{j}(\mathcal{F}) & =\max _{q \in Q(\mathcal{F})} q_{j}^{-1}, j=1, \ldots, n, \\
\theta(\mathcal{F}) & =\left(\frac{\mu(\mathcal{F})}{\mu_{1}(\mathcal{F})}, \ldots, \frac{\mu(\mathcal{F})}{\mu_{n}(\mathcal{F})}\right) .
\end{aligned}
$$

Received by the editors January 8, 2002.

2000 Mathematics Subject Classification. Primary 35B65, 35H10; Secondary 35N10.

Key words and phrases. Systems of differential operators, Newton polyhedron, multi-quasiellipticity, Gevrey vectors, Gevrey spaces, Gevrey regularity.

(C)2002 American Mathematical Society 
Definition 2. Let $\mathcal{F}$ be a regular Newton's polyhedron and $s \in \mathbb{R}_{+}$. We define the generalized Gevrey space $G^{\mathcal{F}, s}(\Omega)$ by the space of $u \in C^{\infty}(\Omega)$ such that $\forall H$ compact of $\Omega, \exists C>0, \forall \alpha \in \mathbb{N}^{n}$,

$$
\sup _{H}\left|D^{\alpha} u\right| \leq C^{|\alpha|+1}[\Gamma(\mu(\mathcal{F}) k(\alpha, \mathcal{F})+1)]^{s},
$$

where $\Gamma$ is the gamma function.

Remark 1. One can take $\sup _{H}\left|D^{\alpha} u\right|$ or $\left\|D^{\alpha} u\right\|_{L^{2}(H)}$ in the definition, according to Sobolev imbedding theorems.

Definition 3. The system $\left(P_{j}\right)_{j=1}^{N}$ is said to be multi-quasi-elliptic in $\Omega$ if

1) The $\mathcal{F}(x)$ do not depend on $x \in \Omega$, i.e. $\forall x, \mathcal{F}(x)=\mathcal{F}$.

2) $\mathcal{F}$ is regular.

3) $\forall x \in \Omega, \exists C>0, \exists R \geq 0, \forall \xi \in \mathbb{R}^{n},|\xi| \geq R$,

$$
\sum_{j=1}^{N}\left|P_{j}(x, \xi)\right| \geq C \sum_{\alpha \in \mathbb{Z}_{+}^{n} \cap \mathcal{F}}\left|\xi^{\alpha}\right| .
$$

Definition 4. Let $\left(P_{j}\right)_{j=1}^{N}$ be a system of linear differential operators satisfying conditions 1) and 2) of Definition 3 and $s \in \mathbb{R}_{+}$, the space of Gevrey vectors of the system $\left(P_{j}\right)_{j=1}^{N}$, denoted $G^{s}\left(\Omega,\left(P_{j}\right)_{j=1}^{N}\right)$, is the space of $u \in C^{\infty}(\Omega)$ such that $\forall H$ compact of $\Omega, \exists C>0, \forall l \in \mathbb{N}, 1 \leq i_{l} \leq N$,

$$
\left\|P_{i_{1}} \ldots P_{i_{l}} u\right\|_{L^{2}(H)} \leq C^{l+1}(l !)^{s \mu(\mathcal{F})} .
$$

The aim of this work is to show the following theorem.

Theorem 1. Let $\Omega$ be an open subset of $\mathbb{R}^{n}, \sigma>s \geq 1$ and $\left(P_{j}\right)_{j=1}^{N}$ be a system of linear differential operators with $G^{\theta(\mathcal{F}), \sigma}(\Omega)$ coefficients. Then

$$
\left(P_{j}\right)_{j=1}^{N} \text { is multi-quasi-elliptic in } \Omega \Longleftrightarrow G^{s}\left(\Omega,\left(P_{j}\right)_{j=1}^{N}\right) \subset G^{\mathcal{F}, s}(\Omega) \text {. }
$$

Some consequences of this theorem are given in section 4 . For differential operators with constant coefficients we have shown in 3] a more general result.

\section{Sufficient CONDITION}

The proof of the sufficient condition follows essentially the work of Zanghirati [6], so we refer for details to this paper.

Instead of $Q(\mathcal{F}), k(\mathcal{F}, \alpha), \mu(\mathcal{F}), \theta(\mathcal{F})$ we write, respectively, $Q, k(\alpha), \mu, \theta$. Denote $\mathcal{K}=\left\{k=k(\alpha): \alpha \in \mathbb{N}^{n}\right\}$. If $\omega$ is an open subset of $\mathbb{R}^{n}, u \in C^{\infty}(\omega)$ and $k \in \mathcal{K}$, define $|u|_{k, \omega}=\sum_{k(\alpha)=k}\left\|D^{\alpha} u\right\|_{L^{2}(\omega)}$. When $u \in C_{0}^{\infty}\left(\mathbb{R}^{n}\right)$ we write $|u|_{k}$.

Let $\left(P_{j}\right)_{j=1}^{N}$ be a system of linear differential operators with coefficients defined in an open neighborhood $\Omega$ of the origin satisfying the following conditions:

(i) The system $\left(P_{j}\right)_{j=1}^{N}$ is multi-quasi-elliptic in $\Omega$.

(ii) The coefficients $a_{j \alpha} \in G^{\theta, s}(\Omega), \forall \alpha \in \mathcal{F}, \forall j \in\{1, \ldots, N\}$.

For $\rho>0$, we denote $B_{\rho}=\left\{x \in \mathbb{R}^{n}, \sum_{j=1}^{n} x_{j}^{2 \mu_{j} / \mu}<\rho^{2}\right\}$. We define for $h \in \mathbb{N}$, $P_{j}^{h}(x, D)=\underbrace{P_{j}(x, D) \circ \cdots \circ P_{j}(x, D)}_{h \text { times }}, j=1, \ldots, N$. 
From the multi-quasi-ellipticity of the system $\left(P_{j}\right)_{j=1}^{N}$ and following the proof of Lemma 3.4 of [6], we obtain

Lemma 1. There exist $\rho_{0}>0$ and $\left.C_{1}>0, \forall \varepsilon \in\right] 0, \frac{1}{v(n)}[(v(n)$ denote the number of elements of $\mathcal{K} \cap\left[0, n[), \exists C_{2}(\varepsilon)>0, \forall \delta \in\right] 0,1\left[, \forall \rho>0, B_{\rho+\delta} \subset B_{\rho_{0}}, \forall u \in\right.$ $C^{\infty}\left(B_{\rho_{0}}\right), \forall p \geq n$,

$$
\begin{aligned}
|u|_{p+1, B_{\rho}} & \leq C_{1}\left(\sum_{j=1}^{N}\left|P_{j}^{n}(x, D) u\right|_{p-n+1, B_{\rho+\delta}}+\varepsilon|u|_{p+1, B_{\rho+\delta}}+(\varepsilon \delta)^{-n \mu}|u|_{p-n+1, B_{\rho+\delta}}\right. \\
& \left.+\sum_{h=0}^{p}\left(\frac{(p+1) !}{h !}\right)^{s \mu} C_{2}(\varepsilon)^{p+1-h}|u|_{h, B_{\rho+\delta}}\right),
\end{aligned}
$$

and for $p \leq n$, we have

$|u|_{p+1, B_{\rho}} \leq C_{1}\left(\sum_{j=1}^{N}\left|P_{j}^{n}(x, D) u\right|_{p-n+1, B_{\rho+\delta}}+\varepsilon|u|_{p+1, B_{\rho+\delta}}+(\varepsilon \delta)^{-(p+1) \mu}|u|_{0, B_{\rho+\delta}}\right)$.

Let $\lambda>0$ and $R>0$. For $p \in \mathbb{N}$, we set

$$
\sigma_{p}(u, \lambda)=(p !)^{-s \mu} \lambda^{-p} \sup _{R / 2 \leq \rho<R}(R-\rho)^{p \mu}|u|_{p, B_{\rho}} .
$$

Lemma 2. Let $\rho_{0}$ be as in the Lemma 1 and let $0<R<1$ such that $\bar{B}_{R} \subset B_{\rho_{0}}$. Then there exists $\lambda_{0}>0\left(\lambda_{0}\right.$ depends only on $R$ and $\left.\left(P_{j}\right)_{j=1}^{N}\right), \forall u \in C^{\infty}\left(B_{\rho_{0}}\right)$, $\forall \lambda \geq \lambda_{0}, \forall p \geq n$

$$
\sigma_{p+1}(u, \lambda) \leq[(p-n+2) \ldots(p+1)]^{-s \mu} \sum_{j=1}^{N} \sigma_{p-n+1}\left(P_{j}^{n} u, \lambda\right)+\sum_{h=0}^{p} \sigma_{h}(u, \lambda),
$$

and for $p \leq n-1$,

$$
\sigma_{p+1}(u, \lambda) \leq(p+1) !^{-s \mu} \sum_{j=1}^{N} \sigma_{0}\left(P_{j}^{p+1} u, \lambda\right)+\sigma_{0}(u, \lambda) .
$$

Proof. Let $p \geq n$, multiply both sides of $(2.1)$ by $(p+1) !^{-s \mu} \lambda^{-p-1}(R-\rho)^{p \mu}$, put $\delta=\frac{R-\rho}{p-n+2}$ and then taking the sup over $\rho \in[R / 2, R[$, we obtain

$$
\sigma_{p+1}(u, \lambda) \leq C_{1}\left(I_{1}+\varepsilon I_{2}+\varepsilon^{-n \mu} I_{3}+I_{4}\right),
$$

where $I_{1}, I_{2}, I_{3}$ and $I_{4}$ are such that

$$
\begin{aligned}
I_{1} & \leq \sum_{j=1}^{N}\left(\frac{(p-n+1) !}{(p+1) !}\right)^{s \mu} \frac{e^{\mu}}{\lambda^{n}} \sigma_{p-n+1}\left(P_{j}^{n} u, \lambda\right), \\
I_{2} & \leq\left(2^{n} e\right)^{\mu} \sigma_{p+1}(u, \lambda) \\
I_{3} & \leq \frac{e^{\mu}}{\lambda^{n}} \sigma_{p-n+1}(u, \lambda), \\
I_{4} & \leq \frac{e^{\mu} C_{2}(\varepsilon)}{\lambda} \sum_{h=0}^{p}\left(\frac{C_{2}(\varepsilon)}{\lambda}\right)^{p-h} \sigma_{h}(u, \lambda) .
\end{aligned}
$$


By a suitable choice of $\varepsilon$, we find

$$
\begin{aligned}
\sigma_{p+1}(u, \lambda) \leq & \left(\frac{(p-n+1) !}{(p+1) !}\right)^{s \mu} \frac{\widetilde{C}_{1}}{\lambda^{n}} \sum_{j=1}^{N} \sigma_{p-n+1}\left(P_{j}^{n} u, \lambda\right)+\frac{\widetilde{C}_{2}}{\lambda^{n}} \sigma_{p-n+1}(u, \lambda) \\
& +\frac{\widetilde{C}_{3}}{\lambda} \sum_{h=0}^{p}\left(\frac{\widetilde{C}_{4}}{\lambda}\right)^{p-h} \sigma_{h}(u, \lambda) .
\end{aligned}
$$

It suffices to take $\lambda_{0}=\widetilde{C}_{1}+\widetilde{C}_{2}+\widetilde{C}_{3}+\widetilde{C}_{4}$ to get (2.3). For the inequality (2.4) we multiply both sides of inequality $(2.2)$ by $\frac{(R-\rho)^{(p+1) \mu}}{(p+1) !^{s \mu} \lambda^{p+1}}$, take $\delta=\frac{R-\rho}{2}$ and then we follow the same procedure for obtaining (2.3).

Lemma 3. Let $\rho_{0}, R$ and $\lambda_{0}$ be as in Lemma 2. Then for any $u \in C^{\infty}\left(B_{\rho_{0}}\right), \forall \lambda \geq$ $\lambda_{0}, \forall p \in \mathbb{N}$, we have

$$
\sigma_{p+1}(u, \lambda) \leq 2^{p+1} \sigma_{0}(u, \lambda)+\sum_{l=1}^{p+1} 2^{p+1-l} C_{p+1}^{l} \frac{1}{(l !)^{s \mu}} \sum_{1 \leq i_{1}, \ldots, i_{l} \leq N} \sigma_{0}\left(P_{i_{1}} \ldots P_{i_{l}} u, \lambda\right) .
$$

Proof. It is obtained by recurrence over $p$.

Our first result is the following theorem, wich generalizes the results of [6], [7] and [8] to systems.

Theorem 2. Let $\Omega$ be an open subset of $\mathbb{R}^{n}, s \geq 1$ and $\left(P_{j}(x, D)\right)_{j=1}^{N}$ be a system of linear differential operators with $G^{\theta, s}(\Omega)$ coefficients. Then

$$
\left(P_{j}\right)_{j=1}^{N} \text { is multi-quasi-elliptic in } \Omega \Rightarrow G^{s}\left(\Omega,\left(P_{j}\right)_{j=1}^{N}\right) \subset G^{\mathcal{F}, s}(\Omega) .
$$

Proof. It is sufficient to check (1.1) in a neighborhood of every point $x$ of $\Omega$. Let us assume $x$ is the origin. Then there exist $\rho_{0}, \lambda_{0}$ and $R$ such that the precedent lemmas hold. Let $u \in G^{s}\left(\Omega,\left(P_{j}\right)_{j=1}^{N}\right)$. Then there is $C_{1}>0$ such that

$$
\sigma_{0}\left(P_{i_{1}} \ldots P_{i_{l}} u, \lambda_{0}\right) \leq C_{1}^{l+1}(l !)^{s \mu}, \forall l \in \mathbb{N}
$$

hence from $(2.5)$, we obtain

$$
\sigma_{p+1}\left(u, \lambda_{0}\right) \leq C_{1}\left(2+N C_{1}\right)^{p+1}, \forall p \in \mathbb{N},
$$

which gives

$$
|u|_{p+1, B_{R / 2}} \leq(p+1) !{ }^{s \mu} C_{2}^{(p+1) \mu+1}, \forall p \in \mathbb{N} .
$$

Following the same steps as in [6] we obtain

$$
|u|_{k, B_{R / 2}} \leq \widetilde{C}^{k \mu+1}(\Gamma(k+1))^{s \mu} .
$$

Consequently from (2.7) it is easy, as in [6], to obtain the estimate (1.1).

\section{NECESSARY CONDITION}

In this section we prove the converse of Theorem 2. For this aim we need a characterization of the multi-quasi-ellipticity of the system $\left(P_{j}(x, D)\right)_{j=1}^{N}$, known in the case of a scalar operator; see [4]. 
Proposition 1. A system $\left(P_{j}\right)_{j=1}^{N}$, satisfying 1) and 2) of Definition 2, is multiquasi-elliptic in $\Omega$ if and only if for any $x \in \Omega, \forall q \in Q$,

$$
\sum_{j=1}^{N}\left|P_{j q}(x, \xi)\right| \neq 0, \quad \forall \xi \in \mathbb{R}^{n}, \xi_{1} \ldots \xi_{n} \neq 0,
$$

where $P_{j q}$ is the q-quasi-homogenous part of $P_{j}$, i.e.

$$
P_{j q}(x, \xi)=\sum_{\langle\alpha, q\rangle=1} a_{j \alpha}(x) \xi^{\alpha} .
$$

Theorem 3. Let $\Omega$ be an open subset of $\mathbb{R}^{n}$ and $P_{j}(x, D), j=1, \ldots, N$, be differential operators with $G^{\theta, \sigma}(\Omega)$ coefficients. If $s>\sigma \geq 1$, then

$$
G^{s}\left(\Omega,\left(P_{j}\right)_{j=1}^{N}\right) \subset G^{\mathcal{F}, s}(\Omega) \Rightarrow\left(P_{j}\right)_{j=1}^{N} \text { is multi-quasi-elliptic in } \Omega \text {. }
$$

Proof. Assume that the system $\left(P_{j}\right)_{j=1}^{N}$ is not multi-quasi-elliptic. Then there exist $x_{0} \in \Omega, q \in Q$ and $\xi_{0} \in S^{n-1}, \xi_{0,1} \ldots \xi_{0, n} \neq 0$, such that

$$
P_{j q}\left(x_{0}, \xi_{0}\right)=0, \quad \forall j=1, \ldots, N .
$$

We construct a function $u \in G^{s}\left(\Omega,\left(P_{j}\right)_{j=1}^{N}\right)$ such that $u \notin G^{\mathcal{F}, s}(\Omega)$, which contradicts the hypothesis. Put $\eta=\frac{1-\varepsilon / \mu}{\mu s}$, and choose $\varepsilon$ satisfying

$$
0<\varepsilon \leq \frac{\mu(s-\sigma)}{2 \mu s-\sigma}<\frac{1}{2} \text { and } \varepsilon<\min _{\langle\beta, q\rangle<1} \mu(1-\langle\beta, q\rangle) .
$$

Let $\delta>0$ such that the ball $B_{0}=B\left(x_{0}, 2 \delta\right)$ is relatively compact in $\Omega$ and $\varphi \in G^{q, \sigma \mu}\left(\mathbb{R}^{n}\right)$ with compact support in $B(0,2 \delta)$ and $\varphi(x) \equiv 1$ in $B(0, \delta)$. The desired function is defined by

$$
u(x)=\int_{1}^{+\infty} \varphi\left[r^{\varepsilon q}\left(x-x_{0}\right)\right] e^{-r^{\eta}} e^{i\left\langle x-x_{0}, r^{q} \xi_{0}\right\rangle} d r,
$$

where $r^{q} x=\left(r^{q_{1}} x_{1}, r^{q_{2}} x_{2}, \ldots, r^{q_{n}} x_{n}\right)$.

Following [5] and [8] it is easy to show that $u \notin G^{\mathcal{F}, s}(U)$ for any neighborhood $U$ of $x_{0}$.

Let us verify that $u \in G^{s}\left(\Omega,\left(P_{j}\right)_{j=1}^{N}\right)$. Since the coefficients of the operators $P_{j}$ are in $G^{\theta, \sigma}(\Omega) \subset G^{q, \sigma \mu}(\Omega)$, then $\exists M>0, \forall \alpha \in \mathbb{Z}_{+}^{n}, \forall \beta \in \mathbb{Z}_{+}^{n}, \forall x \in B_{0}, \forall r \geq 1$, $\forall j=1, \ldots, N$, such that

$$
\left|\left(D_{x}^{\beta} P_{j}^{(\alpha)}\right)\left(x, r^{q} \xi_{0}\right)\right| \leq M^{|\beta|+1}[\Gamma(\langle\beta, q\rangle+1)]^{\sigma \mu} r^{1-\langle\alpha, q\rangle} .
$$

On the other hand in view of (3.1) it is easy to obtain $\forall \delta>0, \exists C_{1}>0, \forall r \geq 1$, $\forall x \in \Omega,\left|x-x_{0}\right|<2 \delta r^{-\varepsilon / \mu}, \forall j=1, \ldots, N$,

$$
\left|P_{j}\left(x, r^{q} \xi_{0}\right)\right| \leq C_{1} r^{1-\varepsilon / \mu} .
$$

Now we need a convenient form of $P_{i_{k}} \ldots P_{i_{1}} u$, for any integer $k \geq 1$. The generalized Leibniz formula $P_{j}(x, D)(u v)=\sum_{\alpha} \frac{1}{\alpha !} P_{j}^{(\alpha)} u D^{\alpha} v$ gives

$$
P_{i_{k}} \ldots P_{i_{0}} u(x)=\int_{1}^{+\infty} A_{i_{k} \ldots i_{0}}(x, r) e^{-r^{\eta}} e^{i\left\langle x-x_{0}, r^{q} \xi_{0}\right\rangle} d r
$$


where $1 \leq i_{l} \leq N$, for any integer $l \leq k, P_{i_{0}}$ designs the identity operator, and

$$
\left\{\begin{array}{c}
A_{i_{0}}(x, r)=\varphi\left[r^{\varepsilon q}\left(x-x_{0}\right)\right] \\
A_{i_{k+1}, i_{k} \ldots i_{0}}(x, r)=\sum_{\langle\alpha, q\rangle \leq 1} \frac{1}{\alpha !} P_{i_{k+1}}^{(\alpha)}\left(x, r^{q} \xi_{0}\right) D_{x}^{\alpha} A_{i_{k} \ldots i_{0}}(x, r) .
\end{array}\right.
$$

To complete the proof we need the following

Lemma 4. $\exists L>0, \exists L_{0}>0, \exists C_{0}>0, \forall k \in \mathbb{Z}_{+}, \forall \gamma \in \mathbb{Z}_{+}^{n}, \forall x \in B_{0}, \forall r \geq 1$,

$$
\begin{aligned}
\left|D_{x}^{\gamma} A_{i_{k} \ldots i_{0}}(x, r)\right| & \leq C_{0}\left(L_{0} r^{\varepsilon}\right)^{\langle\gamma, q\rangle} L^{k}\left(r^{(1-\varepsilon / \mu) k}[\Gamma(\langle\gamma, q\rangle+1)]^{\sigma \mu}\right. \\
& \left.+[\Gamma(\langle\gamma, q\rangle+k+1)]^{\sigma \mu} r^{k \varepsilon(2-1 / \mu)}\right) .
\end{aligned}
$$

Proof. It is obtained by recurrence over $k$. In fact for $k=0$, the estimate (3.5) means $\varphi \in G_{0}^{q, \sigma \mu}\left(\mathbb{R}^{n}\right)$. So suppose that the estimate (3.5) holds up to the order $k$ and let us check it at the order $k+1$. Set $\lambda=r^{1-\varepsilon / \mu}$ and $\tau=r^{\varepsilon(2-1 / \mu)}$. Then the estimate (3.5) is written as

$$
\left|D_{x}^{\gamma} A_{i_{k} \ldots i_{0}}(x, r)\right| \leq C_{0}\left(L_{0} r^{\varepsilon}\right)^{\langle\gamma, q\rangle} L^{k} S(k, \gamma),
$$

where

$$
S(k, \beta)=\lambda^{k}[\Gamma(\langle\beta, q\rangle+1)]^{\sigma \mu}+[\Gamma(\langle\beta, q\rangle+k+1)]^{\sigma \mu} \tau^{k} .
$$

Let $\omega=\min _{1 \leq j \leq n} q_{j}$. Then we have

$$
\lambda^{1-\langle\alpha, q\rangle} \tau^{\langle\alpha, q\rangle} S(k, \beta+\alpha) \leq 2^{\frac{\sigma \mu}{\omega}+1} S(k+1, \beta), \quad\langle\alpha, q\rangle \leq 1 .
$$

From (3.4), we have

$$
\left|D_{x}^{\gamma} A_{i_{k+1} \ldots i_{0}}(x, r)\right| \leq I_{1}+I_{2}+I_{3},
$$

where

$$
\begin{aligned}
I_{1} & =\left|P_{i_{k+1}}\left(x, r^{q} \xi_{0}\right)\right|\left|D_{x}^{\gamma} A_{i_{k} \ldots i_{0}}(x, r)\right|, \\
I_{2} & =\sum_{\beta<\gamma}\left(\begin{array}{l}
\gamma \\
\beta
\end{array}\right)\left|D_{x}^{\gamma-\beta} P_{i_{k+1}}\left(x, r^{q} \xi_{0}\right)\right|\left|D_{x}^{\beta} A_{i_{k} \ldots i_{0}}(x, r)\right|, \\
I_{3} & =\sum_{0<\langle\alpha, q\rangle \leq 1} \sum_{\beta \leq \gamma} \frac{1}{\alpha !}\left(\begin{array}{c}
\gamma \\
\beta
\end{array}\right)\left|D_{x}^{\gamma-\beta} P_{i_{k+1}}^{(\alpha)}\left(x, r^{q} \xi_{0}\right)\right|\left|D_{x}^{\alpha+\beta} A_{i_{k} \ldots i_{0}}(x, r)\right| .
\end{aligned}
$$

Since $A_{i_{k} \ldots i_{0}}$ are functions of compact supports in $B\left(x_{0}, 2 \delta r^{-\varepsilon / \mu}\right)$, and according to (3.3) and (3.6), we have

$$
I_{1} \leq 2^{\frac{\sigma \mu}{\omega}+1} C_{1} C_{0}\left(L_{0} r^{\varepsilon}\right)^{\langle\gamma, q\rangle} S(k+1, \gamma) L^{k} .
$$

The estimates (3.2) and (3.6) give

$$
\begin{gathered}
I_{2} \leq \sum_{\beta<\gamma}\left(\begin{array}{l}
\gamma \\
\beta
\end{array}\right)[\Gamma(\langle\gamma-\beta, q\rangle+1)]^{\sigma \mu} M^{|\gamma-\beta|+1} r^{\varepsilon} C_{0}\left(L_{0} r^{\varepsilon}\right)^{\langle\beta, q\rangle} \\
\cdot 2^{\frac{\sigma \mu}{\omega}+1} S(k+1, \beta) L^{k} .
\end{gathered}
$$

On the other hand, using properties of the gamma function, we have

$$
\left(\begin{array}{l}
\gamma \\
\beta
\end{array}\right)[\Gamma(\langle\gamma-\beta, q\rangle+1)]^{\sigma \mu} S(k, \beta) \leq C_{2}^{\sigma \mu\langle\gamma-\beta, q\rangle} S(k, \gamma) .
$$


Thus we obtain

$$
I_{2} \leq \frac{n M C_{2}^{\sigma \mu}}{L_{0} r^{\varepsilon}} \sum_{\beta \geq 0}\left(\frac{M C_{2}^{\sigma \mu}}{L_{0} r^{\varepsilon}}\right)^{\langle\beta, q\rangle} r^{\varepsilon} 2^{\frac{\sigma \mu}{\omega}+1} M C_{0}\left(L_{0} r^{\varepsilon}\right)^{\langle\gamma, q\rangle} S(k+1, \gamma) L^{k} .
$$

Set $C_{3}=\sum_{\alpha \geq 0}\left(\frac{1}{2}\right)^{\langle\alpha, q\rangle}$, take $L_{0} \geq 2 M C_{2}^{\sigma \mu}$ and $r \geq 1$, and then

$$
I_{2} \leq \frac{n M C_{2}^{\sigma \mu}}{L_{0}} C_{3} 2^{\frac{\sigma \mu}{\omega}+1} M C_{0}\left(L_{0} r^{\varepsilon}\right)^{\langle\gamma, q\rangle} S(k+1, \gamma) L^{k} .
$$

Finally in view of (3.2)

$$
\begin{aligned}
I_{3} \leq & \sum_{0<\langle\alpha, q\rangle \leq 1} \sum_{\beta \leq \gamma}\left(\begin{array}{l}
\gamma \\
\beta
\end{array}\right)[\Gamma(\langle\gamma-\beta, q\rangle+1)]^{\sigma \mu} M^{|\gamma-\beta|+1} r^{1-\langle\alpha, q\rangle} \\
& \times C_{0}\left(L_{0} r^{\varepsilon}\right)^{|\beta+\alpha|} S(k, \beta+\alpha) L^{k} .
\end{aligned}
$$

For any $\alpha \in Z_{+}^{n}, 0<\langle\alpha, q\rangle \leq 1$, we have $r^{1-\langle\alpha, q\rangle+\varepsilon\langle\alpha, q\rangle} \leq \lambda^{1-\langle\alpha, q\rangle} \tau^{\langle\alpha, q\rangle}$, which gives, with (3.6) and (3.8),

$$
I_{3} \leq \sum_{0<\langle\alpha, q\rangle \leq 1} \sum_{\beta \leq \gamma}\left(\frac{M C_{2}^{\sigma \mu}}{L_{0} r^{\varepsilon}}\right)^{|\gamma|} 2^{\frac{\sigma \mu}{\omega}+1} M C_{0} L_{0}^{|\alpha|}\left(L_{0} r^{\varepsilon}\right)^{|\gamma|} S(k+1, \gamma) L^{k} .
$$

Put $C_{4}=\sum_{0<\langle\alpha, q\rangle \leq 1} L_{0}^{\langle\alpha, q\rangle}$. Then we obtain

$$
I_{3} \leq 2^{\frac{\sigma \mu}{\omega}+1} M C_{4} C_{3} C_{0}\left(L_{0} r^{\varepsilon}\right)^{\langle\gamma, q\rangle} S(k+1, \gamma) L^{k} .
$$

If we choose

$$
L \geq 2^{\frac{\sigma \mu}{\omega}+1}\left(C_{1}+\frac{n M^{2} C_{2}^{\sigma \mu}}{L_{0}} C_{3}+M C_{3} C_{4}\right),
$$

we get, from $(3.7),(3.9)$ and $(3.10)$,

$$
I_{1}+I_{2}+I_{3} \leq C_{0}\left(L_{0} r^{\varepsilon}\right)^{\langle\gamma, q\rangle} S(k+1, \gamma) L^{k+1},
$$

which means that (3.5) holds at the order $k+1$.

End of the Proof of Theorem 3. Applying the last lemma for $\gamma=0$, we find

$$
\left|A_{i_{k} \ldots i_{0}}(x, r)\right| \leq C_{0}^{\prime} L^{k}\left(r^{(1-\varepsilon / \mu) k}+(k !)^{\sigma \mu} r^{k \varepsilon(2-1 / \mu)}\right) .
$$

Thus we obtain

$$
\left|A_{i_{k} \ldots i_{0}}(x, r)\right| \leq C_{0}^{\prime} L^{k}(2 s \mu)^{k \mu s}(k !)^{s \mu}\left[\exp \left(\frac{r^{\eta}}{2}\right)+\exp \left(\frac{r^{\eta^{\prime}}}{2}\right)\right],
$$

where $\eta^{\prime}=\frac{\varepsilon(2-1 / \mu)}{\mu(s-\sigma)} \leq \eta=\frac{1-\varepsilon / \mu}{\mu s}$, since $\varepsilon \leq \frac{\mu(s-\sigma)}{2 \mu s-\sigma}$.

Therefore

$$
\begin{aligned}
\left|P_{i_{k} \ldots} P_{i_{0}} u(x)\right| & \leq 2 C_{0}^{\prime} L^{\prime k}(k !)^{s \mu} \int_{1}^{+\infty} \exp \left(-\frac{r^{\eta}}{2}\right) d r \\
& \leq C^{k+1}(k) !^{s \mu}
\end{aligned}
$$

which means that $u \in G^{s}\left(\Omega,\left(P_{j}\right)_{j=1}^{N}\right)$. 


\section{Consequences}

A first consequence of Theorem 2 is a result on Gevrey-hypoellipticity for multiquasi-elliptic systems.

Corollary 1. Under the assumptions of Theorem 2, the following propositions are equivalent:

(i) $u \in \mathcal{D}^{\prime}(\Omega), P_{j} u \in G^{\mathcal{F}, s}(\Omega), \forall j=1, \ldots, N$.

(ii) $u \in G^{\mathcal{F}, s}(\Omega)$.

The theorems of this work unify the results of Bolley-Camus [1] and Métivier [5] in the homogenous case, the results of Zanghirati [7] and [8] in the scalar quasihomogenous case and generalize them to quasi-homogenous systems.

Corollary 2. Let $\Omega$ be an open subset of $\mathbb{R}^{n}$ and $\sigma>s \geq 1$, and let $\left(P_{j}\right)_{j=1}^{N}$ be a system of linear differential operators with coefficients in $G^{q, \sigma}(\Omega)$. Then

$$
\left(P_{j}\right)_{j=1}^{N} \text { is q-quasi-elliptic in } \Omega \Longleftrightarrow G^{s}\left(\Omega,\left(P_{j}\right)_{j=1}^{N}\right) \subset G^{q, s}(\Omega) \text {. }
$$

\section{REFERENCES}

[1] P. Bolley, J. Camus, Powers and Gevrey regularity for a system of differential operators, Czechoslovak Math. J., Praha, 29 (104), (1979), 649-661. MR 80i:35043

[2] P. Bolley, J. Camus, L. Rodino, Hypoellipticité analytique-Gevrey et itérés d'opérateurs, Ren. Sem. Mat. Univ. Politec. Torino, vol. 45:3, (1989), 1-61. MR 91f:35073

[3] C. Bouzar, R. Chaili, Une généralisation de la propriété des itérés, Arch. Math., 76:1, (2001), 57-66. MR 2001m:35052

[4] S.G. Gindikin, L.R. Volevich, The method of Newton's polyhedron in the theory of partial differential equations, Kluwer Acad. Publ., 1992. MR 95a:35001

[5] G. Métivier, Propriété des itérés et ellipticité, Comm. Partial. Differential Equations, 3, 9, (1978), 827-876. MR 80f:35047

[6] L. Zanghirati, Iterati di una classe di operatori ipoellittici e classi generalizzate di Gevrey, Boll. U.M.I., vol. 1, suppl., (1980), 177-195. MR 84j:35044

[7] L. Zanghirati, Iterati di operatori quasi-ellittici e classi di gevrey, Bollettino U.M.I., 5, 18-B, (1981), 411-428. MR 83a:35024

[8] L. Zanghirati, Complementi al teorema degli iterati quasi-ellitici, Bollettino U.M.I., 6, 1-A, (1982), 137-143. MR 83i:35083

Département de Mathématiques, Université d'Oran Esenia, Oran, Algeria

E-mail address: bouzarchikh@hotmail.com

Département de Mathématiques, U.S.T.O., Oran, Algeria

E-mail address: chaili@mail.univ-usto.dz 